\title{
Glutamate Uptake Disguises Neurotoxic Potency of Glutamate Agonists in Cerebral Cortex in Dissociated Cell Culture
}

\author{
Paul A. Rosenberg, Snehal Amin, and Melanie Leitner \\ Department of Neurology and Program in Neuroscience, Children's Hospital and Harvard Medical School, Boston, \\ Massachusetts 02115
}

\begin{abstract}
The pharmacological properties of glutamate agonists were compared in astrocyte-rich and astrocyte-poor cultures derived from embryonic rat cerebral cortex. The object of this investigation was to determine the extent to which glutamate uptake might influence the receptor-mediated neurotoxic actions of these compounds. In astrocyte-rich cultures, using $30 \mathrm{~min}$ exposures, we observed that the potencies of the poorly transported agonists NMDA $(35 \mu \mathrm{M})$ and $\mathrm{D}$-glutamate $(89 \mu \mathrm{M})$ were higher than that of L-glutamate $(205 \mu \mathrm{M})$. In astrocyte-poor cultures, L-glutamate was much more potent, with an $E C_{50}$ of $5 \pm 4 \mu \mathrm{M}(3-12 \mu \mathrm{M})$, for a $30 \mathrm{~min}$ exposure, whereas the potencies of NMDA and D-glutarnate were essentially unchanged. $L-$ and $D$-aspartate were also more effective in astrocyte-poor cultures, again with $\mathrm{EC}_{50}$ values of approximately 6-10 $\mu \mathrm{M}$, as compared with 130 and $108 \mu \mathrm{M}$, respectively, in astrocyte-rich cultures. In other experiments, blocking sodium-dependent glutamate uptake in astrocyterich cultures, by using a sodium-free medium, made glutamate as potent an agonist as in astrocyte-poor cultures. Finally, we directly assessed the glutamate uptake system in astrocyte-rich and astrocyte-poor cultures and found that uptake was reduced approximately 25 -fold in the astrocytepoor cultures. These results show that in the presence of abundant astrocytes the neurotoxic potencies of L-glutamate, L-aspartate, and D-aspartate are substantially underestimated.
\end{abstract}

Glutamate neurotoxicity has been proposed as an important mechanism of neuronal injury and death both in acute catastrophic events such as stroke (Choi and Rothman, 1990) and trauma (Faden et al., 1989; Tecoma et al., 1989) and in the neuropathological changes associated with chronic neurodegenerative diseases as well (Meldrum and Garthwaite, 1990). The cellular mechanisms underlying glutamate receptor-mediated neuronal injury and death are not well understood, and the pharmacology of glutamate neurotoxicity is central to our understanding of these mechanisms. Identification of the critical role played by the NMDA receptor, for example, has been im-

\footnotetext{
Received May 14, 1991; revised July 29, 1991; accepted July 31, 1991.

We are grateful to Sara Vasquez and Can Zhong for excellent technical assistance, to Mai Nguyen-Huynh and Virginia Zaleskas for cell counting, and to Drs. Elias Aizenman, Kristen Harris, and Stuart Lipton for helpful discussions. P.A.R. is supported by grants from the Joseph P. Kennedy, Jr. Foundation, the American Heart Association, and the National Institutes of Health (NS 26830) and by a Mental Retardation CORE Grant to Children's Hospital

Correspondence should be addressed to Dr. Paul A. Rosenberg, Enders Research Building, Department of Neurology, Children's Hospital, 300 Longwood Avenue, Boston, MA 02115

Copyright (C) 1992 Society for Neuroscience $0270-6474 / 92 / 120056-06 \$ 05.00 / 0$
}

portant in establishing a role for calcium in glutamate toxicity (Choi and Rothman, 1990). However, the pathogenic mechanisms that determine when glutamate receptor stimulation will have toxic consequences remain obscure. The intrinsic sensitivity of central neurons to glutamate toxicity is obviously of central importance to understanding the pathogenesis of glutamate receptor-mediated neuronal death.

Previous experiments determined that cortical neurons in tissue culture with reduced numbers of astrocytes present were 100 times more sensitive to a long (18-24 hr) exposure to glutamate than in astrocyte-rich cultures (Rosenberg and Aizenman, 1989). Glutamate toxicity in astrocyte-poor cultures, as in astrocyte-rich cultures (Choi et al., 1988), was mediated by NMDA receptors, and yet no difference was observed in the membrane response of neurons cultured under the two sets of conditions to application of NMDA using whole-cell patchclamp recording techniques. These observations suggested two possible explanations: (1) that differences between the two culture systems with respect to access to NMDA receptors on dendrites might explain the difference in apparent potency of glutamate (Garthwaite, 1985) and (2) that differences in the consequences of NMDA receptor stimulation might account for the difference in potency. An example of the latter mechanism is suggested by previous experiments showing the importance of the sodium-calcium exchange in glutamate toxicity in hippocampal cultures (Mattson et al., 1989a). Differences in the potency of glutamate might be due to differences in the capacity to handle a calcium load in neurons maintained with or without abundant astrocytes (Mattson et al., 1989b).

The current experiments were undertaken to test the hypothesis that glutamate uptake in astrocyte-rich cultures significantly alters the pharmacology of glutamate neurotoxicity in these cultures.

\section{Materials and Methods}

Tissue culture. Dissociated cell cultures derived from rat embryonic cerebral cortex were prepared as described previously (Rosenberg, 1991). Tissue was removed from embryonic day $16 \mathrm{CD}$ rat fetuses, dissociated using trypsin, and plated on poly-L-lysine- or collagen plus poly-Llysine-coated glass coverslips. Astrocyte-rich cultures were grown in medium containing Dulbecco's modified Eagle's medium (DMEM), Ham's F-12, and calf serum (DHS; $8: 1: 1$ ) containing $2 \mathrm{~mm}$ glutamine, penicillin and streptomycin, $25 \mathrm{~mm}$ HEPES, and $25 \mathrm{~mm}$ glucose. At 15 $\mathrm{d}$ in vitro, these cultures were exposed to $5 \mu \mathrm{M}$ cytosine arabinoside for $72 \mathrm{hr}$. Astrocyte-poor cultures were grown in DHS for $4 \mathrm{~d}$, at which point they were exposed to cytosine arabinoside for $48 \mathrm{hr}$. On day 7 , medium was removed and replaced with a serum-free medium containing minimum essential medium (MEM), DMEM, and Ham's F-12 (5:4:1) with the N2 additives used by Bottenstein and Sato (1979), in addition to catalase (Walicke ct al., 1986), penicillin and streptomycin, $2 \mathrm{~mm}$ glutamine, and $11 \mathrm{~mm}$ glucose. Medium was not subsequently 
changed in astrocyte-poor cultures, and they were placed on watersoaked filter paper pads to retard loss of water from the culture medium.

Toxicity experiments. Procedures were used that have been previously described (Rosenberg and Aizenman, 1989; Rosenberg, 1991). Astrocyte-rich cultures were washed once with physiological saline and then placed in wells containing MEM, plus selected concentrations of drugs. Wells were incubated for $30 \mathrm{~min}$ at $35^{\circ} \mathrm{C}$ in $5 \% \mathrm{CO}_{2}$ and, for most experiments, were gently agitated during this period. After exposure to drugs, medium was replaced with fresh medium, and cultures were returned to the incubator for 20-24 hr. Experiments with astrocyte-rich cultures were terminated by staining with trypan blue and fixing in $2.5 \%$ glutaraldehyde (Pixley and Cotman, 1985; Rosenberg, 1988). Experiments with astrocyte-poor cultures were terminated in most experiments by fixation in $2.5 \%$ glutaraldehyde, since these cultures are damaged by repeated washing, and because dead cells in these cultures rapidly disintegrate, leaving few to pick up the trypan blue stain (Rosenberg and Aizenman, 1989). Surviving neurons were counted in 10 fields per coverslip, using phase-contrast microscopy at $240 \times$ magnification.

In sodium substitution experiments, the exposure medium was Trisbuffered saline (Choi, 1987) using sodium chloride, choline chloride, or lithium chloride.

Astrocyte-poor cultures were used at $18-40 \mathrm{~d}$ in vitro. Astrocyte-rich cultures were used at $27-68 \mathrm{~d}$ in vitro. For D-glutamate, three and four culture dates (individual platings) were used for the reported experiments with astrocyte-poor and astrocyte-rich cultures, respectively; for D- and L-aspartate, two and four culture dates were used for experiments with astrocyte-poor and astrocyte-rich cultures, respectively; three different dates were used for the L-glutamate experiments with astrocyterich cultures, seven for the L-glutamate experiments with astrocyte-poor cultures; three different dates for the NMDA experiments with both culture types; seven different dates for the sodium depletion experiments with astrocyte-rich cultures and four with astrocyte-poor cultures; and two different dates for the sodium depletion experiments with lithium replacement. Experiments were performed at least in duplicate and usually in triplicate or quadruplicate (Rosenberg, 1991).

Uptake experiments. The method of Drejer et al. (1985) was used for experiments, with the exception that the medium was MEM, the same as that for the toxicity experiments. ${ }^{3} \mathrm{H}$-D-aspartate (Amersham TRK.66, batch 23 ; specific activity, $33 \mathrm{Ci} / \mathrm{mmol}$; adjusted to $0.5 \mu \mathrm{Ci} / \mathrm{nmol}$ with nonradioactive $\mathrm{D}$-aspartate) was used as the substrate for measuring uptake. After $5 \mathrm{~min}$ exposure, medium was taken and assayed for radioactivity by the method of liquid scintillation. Counting efficiency was approximately $30 \%$. Tissue was digested either with $2 \mathrm{~m} \mathrm{KOH}$ or with $0.1 \mathrm{M} \mathrm{NaOH}$. Tissue samples were assayed for protein using the protein-dye binding method (Bradford, 1976) and a bovine serum albumin standard.

\section{Results}

The neurotoxic potencies of agonists that are and are not substrates for the glutamate uptake system were determined in astrocyte-rich and astrocyte-poor cultures. This allows for a direct test of the hypothesis that glutamate uptake is necessary for the difference in potency of glutamate as a neurotoxin in the two culture systems, exploiting the differences in the affinities for the glutamate uptake carrier of glutamate agonists. Brew and Attwell (1987) have shown the relative magnitude of inward currents evoked via the uptake carrier in retinal glial cells by L-glutamate, L-aspartate, D-aspartate, NMDA, and D-glutamate to be $1: 0.43: 0.25: 0.06: 0$. The hypothesis predicts that transported agonists such as L-glutamate and D- and L-aspartate should be more potent in astrocyte-poor cultures than in astrocyte-rich cultures, whereas weakly transported agonists such as D-glutamate or NMDA should show similar neurotoxic potencies in the two culture systems.

Figure $1 A$ shows a typical dose-response experiment in which astrocyte-rich or astrocyte-poor cultures were exposed to L-glutamate for $30 \mathrm{~min}$ and then returned to MEM for 20-24 hr. As has been reported before, there was a marked increase in potency of glutamate as a neurotoxin in astrocyte-poor cultures. In contrast, Figure $1 B$ shows a similar experiment that used the poorly
A

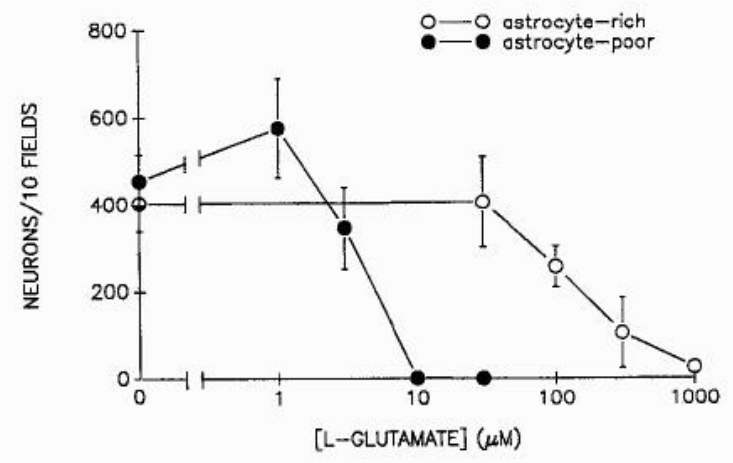

B

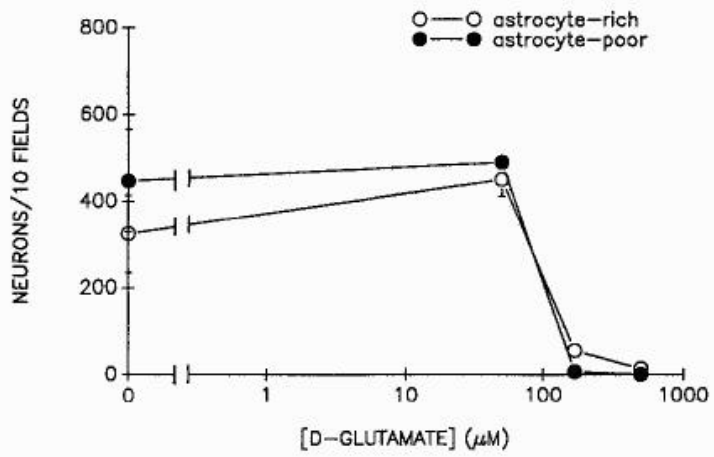

Figure 1 . Neurotoxicity of L-glutamate and D-glutamate in astrocytepoor and in astrocyte-rich cultures. $A$. The dose-response relationship of L-glutamate in astrocyte-poor ( $20 \mathrm{~d}$ in vitro) and in astrocyte-rich ( 34 d in vitro) cultures was determined. Cultures were exposed to agonist for $30 \mathrm{~min}$ in MEM, followed by $20-24 \mathrm{hr}$ in MEM. The potency of $\mathrm{L}$-glutamate in the astrocyte-poor cultures was approximately 34 times that in the astrocyte-rich cultures in this experiment. Error bars indicate $\mathrm{SD}$. $B$, The dose-response relationship of D-glutamate in astrocyte-poor $(19 \mathrm{~d}$ in vitro) and in astrocyte-rich $(27 \mathrm{~d}$ in vitro) cultures. The potency of D-glutamate in the two culture systems was virtually the same.

transported agonist D-glutamate (Drejer et al., 1982; Brew and Attwell, 1987). There was a similar response to this agonist in the two culture systems. D- and L-aspartate are both substrates for the glutamate transporter (Drejer et al., 1982; Brew and Attwell, 1987). Both of these agonists showed a significant enhancement of neurotoxic potency in astrocyte-poor cultures (Fig. 2).

The neurotoxic potencies of the weakly transported agonist NMDA in the two culture systems were compared (Fig. 3). The $\mathrm{EC}_{\text {so }}$ values in the two culture systems were virtually identical. Table 1 summarizes all the data obtained with these agonists and includes, for comparison, data obtained from electrophysiological experiments (Patneau and Mayer, 1990) and binding experiments (Olverman et al., 1988), as well as from previously reported studies using conventional astrocyte-rich murine cortical cultures (Koh and Choi, 1988; Choi et al., 1989).

Further support for the uptake hypothesis comes from experiments involving blockade of sodium-dependent glutamate uptake by removal of extracellular sodium (Fig. $4 A, B$; Table 2). Substitution of choline for sodium increased the potency of glutamate as a neurotoxin in astrocyte-rich cultures by approximately 100 -fold (Fig. 4A, Table 2), but had no significant effect on the potency of glutamate in astrocyte-poor cortical cultures (Fig. $4 B$, Table 2). Substitution of choline for sodium thereby 
A

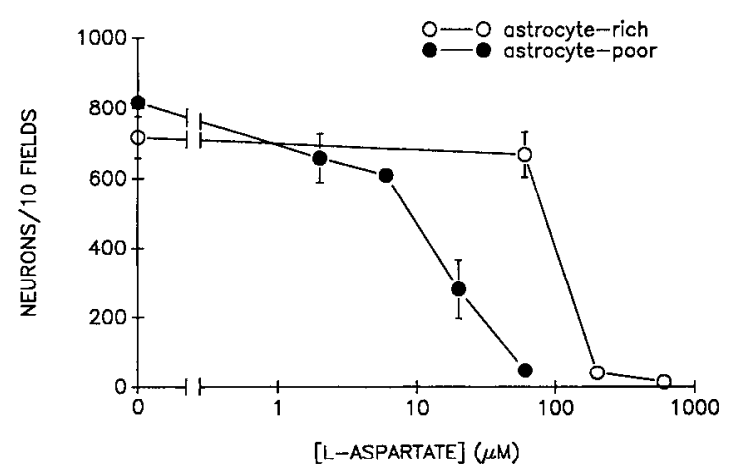

B

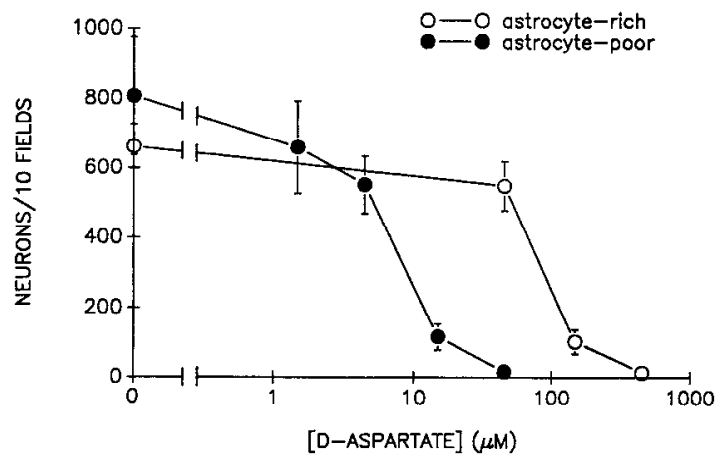

Figure 2. Neurotoxicity of $\mathbf{L}$-aspartate and D-aspartate in astrocytepoor and in astrocyte-rich cultures. $A$, The dose-response relationship of L-aspartate in astrocyte-rich ( $35 \mathrm{~d}$ in vitro) and in astrocyte-poor (18 $\mathrm{d}$ in vitro) cultures. The potency of $\mathrm{L}$-aspartate in astrocyte-poor cultures was approximately 9 times that in astrocyte-rich cultures. $B$, The doseresponse relationship of $D$-aspartate in astrocyte-rich $(35 \mathrm{~d}$ in vitro) and in astrocyte-poor ( $18 \mathrm{~d}$ in vitro) cultures. The potency of D-aspartate in astrocyte-poor cultures was approximately 13 times that in astrocyterich cultures.

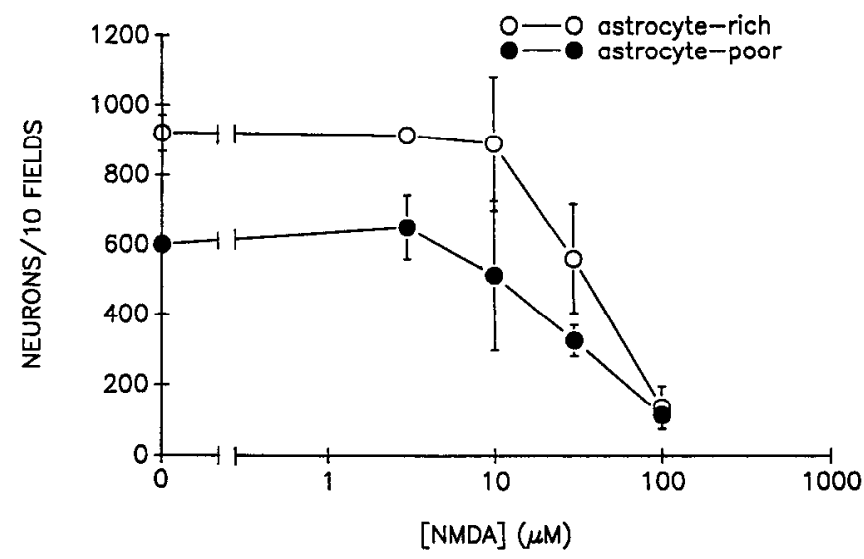

Figure 3. Neurotoxicity of NMDA in astrocyte-poor and in astrocyterich cultures. The dose-response relationship of NMDA in astrocyterich ( $28 \mathrm{~d}$ in vitro) and in astrocyte-poor $(21 \mathrm{~d}$ in vitro) cultures. The $\mathrm{EC}_{50}$ was $37 \mu \mathrm{M}$ in astrocyte-rich cultures and $27 \mu \mathrm{M}$ in astrocyte-poor cultures.

eliminated the difference in the potency of glutamate in the two culture systems and made astrocyte-rich cultures resemble astrocyte-poor cultures. Similarly, substitution of lithium for sodium also increased the potency of glutamate in astrocyte-rich cultures (14-fold; Table 2).

The data presented so far can be explained best by the operation, in the astrocyte-rich cultures, of a glutamate uptake system that is substantially reduced in the astrocyte-poor cultures. We therefore sought to measure this function directly in the two culture systems using the nonmetabolizable substrate D-aspartate (Gordon and Balazs, 1983), according to the method of Drejer et al. (1985). At zero time, ${ }^{3} \mathrm{H}$-D-aspartate was added to a final concentration of $1 \mu \mathrm{M}$, and media and tissue samples were collected after $5 \mathrm{~min}$. The results are shown in Table 3 . When the data were normalized on the basis of cell protein, the uptake measured in the astrocyte-rich cultures, which are 90

\begin{tabular}{|c|c|c|c|c|c|c|}
\hline \multirow[b]{2}{*}{ Agonist } & \multirow{2}{*}{$\begin{array}{l}\text { Rat AP } \\
30 \mathrm{~min}\end{array}$} & \multirow{2}{*}{$\begin{array}{l}\text { Rat AR } \\
30 \text { min }\end{array}$} & \multirow[b]{2}{*}{ Phys $^{a}$} & \multirow[b]{2}{*}{ Binding $^{b}$} & \multicolumn{2}{|c|}{ Mouse AR } \\
\hline & & & & & $5 \min ^{c, d}$ & $24 \mathrm{hr}^{d}$ \\
\hline L-Glutamate & $\begin{array}{c}5 \pm 4 \\
(n=5)\end{array}$ & $\begin{array}{l}205 \pm 103 \\
(n=4)\end{array}$ & 1.1 & 0.9 & 190 & 170 \\
\hline D-Glutamate & $\begin{array}{l}108 \pm 23 \\
(n=5)\end{array}$ & $\begin{array}{l}89 \pm 28 \\
(n=7)\end{array}$ & - & 49 & - & - \\
\hline L-Aspartate & $\begin{array}{c}10 \pm 4 \\
(n=3)\end{array}$ & $\begin{array}{l}130 \pm 46 \\
(n=7)\end{array}$ & 7.5 & 11 & $130-190$ & - \\
\hline D-Aspartate & $\begin{array}{c}6+2 \\
(n=3)\end{array}$ & $\begin{array}{l}108 \pm 30 \\
(n=6)\end{array}$ & - & 10 & $130-190$ & - \\
\hline NMDA & $\begin{array}{l}36 \pm 23 \\
(n=4)\end{array}$ & $\begin{array}{l}35 \pm 10 \\
(n=4)\end{array}$ & 16.4 & 11 & 120 & 16 \\
\hline
\end{tabular}

Data are mean $\mathrm{EC}_{50}$ values $(\mu \mathrm{M})$ for glutamate agonists in astrocyte-rich and astrocyte-poor cultures (30 min exposure) followed by 20-24 hr incubation in MEM. Data are given for rat astrocyte-rich (Rat AR) and rat astrocyte-poor (Rat AP) cortical cultures (present study), electrophysiological experiments in hippocampus (Phys), radioligand binding experiments (Binding), and murine astrocyte-rich cultures (Mouse AR) following $5 \mathrm{~min}$ or $24 \mathrm{hr}$ exposure to agonists.

a Physiology data from Patneau and Mayer (1990).

${ }^{b}$ Binding data from Olverman et al. (1988) using ${ }^{3} \mathrm{H}-\mathrm{APV}$ as the radioligand.

c Neurotoxicity data from Choi et al. (1989).

${ }^{d}$ Neurotoxicity data from Koh and Choi (1988). 
A

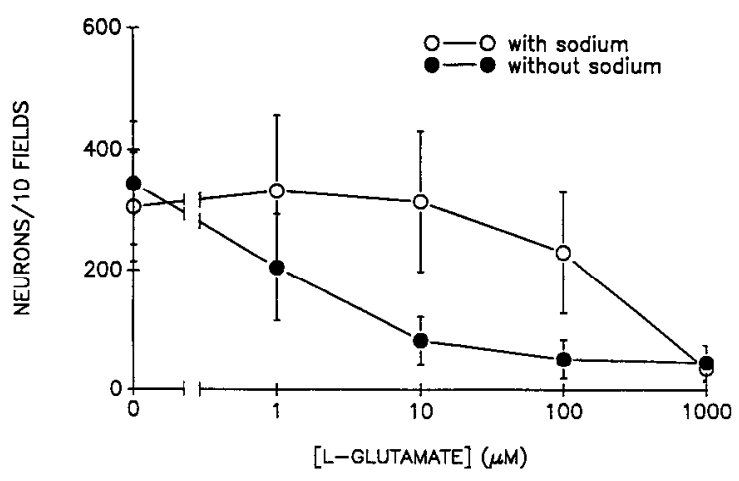

B

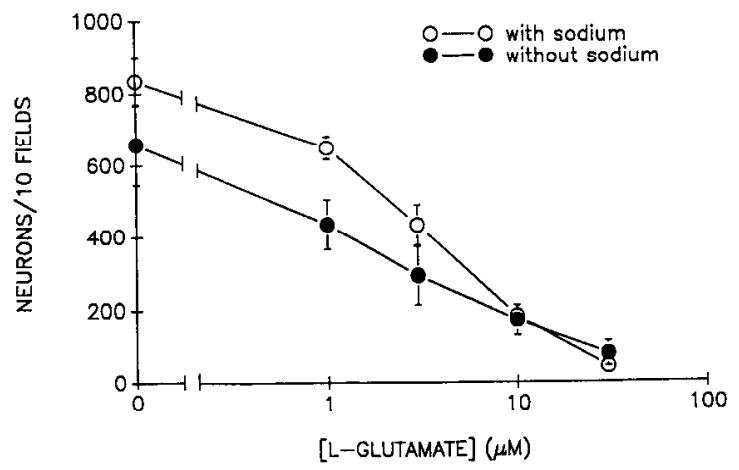

Figure 4. Neurotoxicity of glutamate in astrocyte-rich and astrocytepoor cultures in the presence and absence of sodium. $A$, The doseresponse relationship of $\mathbf{L}$-glutamate in astrocyte-rich cultures was determined in the presence and absence of sodium. The data shown are pooled from seven experiments and represent means \pm SDs. For each experiment, cultures from the same plating were exposed to selected concentrations of glutamate for $30 \mathrm{~min}$ in Tris-buffered saline with or without sodium chloride. In saline without sodium chloride, this salt was replaced by choline chloride. Cultures ranged from 41 to $68 \mathrm{~d}$ in vitro (mean, $58 \mathrm{~d}$ ). $B$, The dose-response relationship of $\mathrm{L}$-glutamate in astrocyte-poor cultures in the presence and absence of sodium. In this experiment, $21 \mathrm{~d}$ in vitro cultures were used. The results shown are from a single experiment, performed in duplicate for each ionic condition.

$95 \%$ astrocytes, was similar to what has been measured previously for astrocyte cultures. When the data were normalized on the basis of surface area covered by the culture, which is the most relevant measure for the purposes of the present study, the astrocyte-poor cultures demonstrated a 25 -fold reduction in uptake [consistent with the fact that per unit area of culture there is approximately $1 / 10$ the protein in astrocyte-poor cultures $\left(10 \mu \mathrm{g} /\right.$ coverslip $\left.=113 \mathrm{~mm}^{2}\right)$ compared to astrocyte-rich cultures (100 $\mu \mathrm{g} /$ coverslip)].
Table 3. Uptake of ${ }^{3} \mathrm{H}-\mathrm{n}$-aspartate into astrocyte-rich and astrocytepoor cultures

\begin{tabular}{lll} 
& \multicolumn{2}{l}{ Aspartate uptake } \\
\cline { 2 - 3 } Culture & $\mathrm{nmol} / \mathrm{mg}$-min & $\begin{array}{l}\mathrm{pmol} / 100 \\
\mathrm{~mm}^{2} \text {-min }\end{array}$ \\
\hline Astrocyte-poor & $0.21 \pm 0.04$ & $1.67 \pm 0.27$ \\
Astrocyte-rich & $0.49 \pm 0.06$ & $42.3 \pm 3.5$ \\
Astrocyte $^{a}$ & $0.53 \pm 0.02$ & -
\end{tabular}

The operation of glutamate uptake systems in the two culture types was assessed using the nonmetabolizable tracer ${ }^{3} \mathrm{H}$-D-aspartate at $1 \mu \mathrm{M}$ with a $5 \mathrm{~min}$ incubation. Uptake rates were calculated from the radioactivity depleted from the media and from the known specific radioactivity in the media. The results are means \pm SEM of five individual experiments.

${ }^{a}$ For comparison the data provided by Drejer et al. (1985) for cultures of astrocytes are also included.

\section{Discussion}

These data provide strong support for the hypothesis that glutamate uptake, presumably by astrocytes, is required for the apparent reduction in potency of glutamate as a neurotoxin in astrocyte-rich cultures. In astrocyte-rich cultures, the order of potency of the glutamate agonists we tested was NMDA > D-glutamate $\approx$ D-aspartate $\approx$ L-aspartate $>$ L-glutamate. This contrasts with the order of the potency series drawn from binding studics using ${ }^{3} \mathrm{H}$-aminophosphonovaleric acid (APV) (Olverman et al., 1988): L-glutamate $>$ L-aspartate $\approx$ D-aspartate $\approx$ NMDA $>$ D-glutamate. The potency series of glutamate toxicity in astrocyte-rich cultures is also different from that obtained using electrophysiological responses in hippocampus (Patneau and Mayer, 1990): L-glutamate > L-aspartate > NMDA. In contrast, neurotoxicity studies in astrocyte-poor cultures yield a potency series compatible with the data obtained by binding and electrophysiological studies: L-glutamate $\approx$ D-aspartate $\approx$ L-aspartate $>$ NMDA $>$ D-glutamate. Of note, however, in the neurotoxicity studies, the potencies of L-glutamate and the stereoisomers of aspartate were similar, whereas the binding and physiology studies show a 7-11-fold higher potency of L-glutamate compared to aspartate.

The present results are consistent with those obtained by Garthwaite (1985), who compared the potency of glutamate agonists in cercbellar slices and in cell suspensions and showed that glutamate uptake disguises the pharmacology of glutamate agonist-stimulated cGMP accumulation in cerebellar slices. A diffusion model was developed to account for these results and predicted that, for any amino acid transported at the same rate as L-glutamate, and whose maximal response occurs at $\leq 100$ $\mu \mathrm{M}$, an external concentration of approximately $1 \mathrm{mM}$ would be required to produce a maximal effect, with an $\mathrm{EC}_{50}$ of approx-

Table 2. The effect of sodium depletion on the potency of glutamate in astrocyte-rich and astrocytepoor cultures

\begin{tabular}{llll} 
& \multicolumn{2}{l}{ Glutamate potency } & \\
\cline { 2 - 4 } Culture & with sodium & with choline & with lithium \\
\hline Astrocyte-poor & $3.6 \pm 2 \quad(n=5)$ & $2.2 \pm 0.9(n=5)$ & - \\
Astrocyte-rich & $269 \pm 189(n=9)$ & $2.6 \pm 2.7(n=7)$ & $19 \pm 10(n=3)$
\end{tabular}

The neurotoxicity of glutamate ( $30 \mathrm{~min}$ exposure) in astrocyte-rich and astrocyte-poor cultures was compared with and without sodium present in the extracellular medium. The data presented are $\mathrm{EC}_{50}$ values $(\mu \mathrm{M})$ for glutamate under the specified ionic and culture conditions. 
imately $300 \mu \mathrm{M}$, regardless of the actual (intrinsic) potency of the amino acid. These parameters (maximal effect, $1 \mathrm{~mm} ; \mathrm{EC}_{30}$, $\approx 300 \mu \mathrm{M}$ ) fit well with those observed for the neurotoxicity of L-glutamate in astrocyte-rich cultures in this and other studies (Koh and Choi, 1988), which, by implication, may be assumed therefore to be largely determined by the transport properties of L-glutamate in astrocyte-rich cultures rather than the interaction of L-glutamate with its receptors. In contrast with Garthwaite's observations in the cerebellar preparations, we observed no differences in the potency of D-glutamate in astrocyte-poor and astrocyte-rich cultures. This suggests that the effects we observed were determined only by the high-affinity and not the low-affinity glutamate uptake system because D-glutamate is a substrate for the low-affinity uptake system (Benjamin and Quastel, 1976).

The hypothesis that glutamate uptake is responsible for the protection against glutamate toxicity observed in the astrocyterich cultures is further supported by the sodium replacement experiments. Substitution of choline for sodium resulted in a marked increase in the potency of $\mathrm{L-glutamate}$ in astrocyte-rich cultures without significantly affecting the potency of glutamate in the astrocyte-poor cultures. This argues strongly for sodiumdependent glutamate uptake as the primary mechanism determining the relative resistance to glutamate toxicity observed in the astrocyte-rich cultures. Since it has been proposed that the sodium-calcium exchange might have a protective role in circumstances that would challenge the ability of neurons to dispose of a calcium load (Mattson et al., 1989), one might imagine an alternative explanation. The absence of a functional sodiumcalcium exchange system in neurons in the astrocyte-poor cultures might increase the neuronal vulnerability to glutamate seen in these cultures. Removal of sodium would inactivate the sodium-calcium exchange in astrocyte-rich cultures, rendering them more sensitive to glutamate, but would have no effect in the astrocyte-poor cultures. However, the similar potency of NMDA as a neurotoxin in the astrocyte-rich and astrocyte-poor cultures suggests that there is no difference in the intrinsic sensitivity of cortical neurons maintained in the two culture systems to NMDA receptor-mediated toxicity.

The influence of growth conditions on sensitivity to excitotoxins is of concern, because the two culture types used for this work were maintained by different methods, necessitated by the difficulty in growing cortical neurons with few astrocytes present. This might conceivably result in a difference in intrinsic vulnerability to excitotoxicity, for example, if the astrocyte-poor culture conditions resulted in an increased expression of NMDA receptors on cortical neurons. We have addressed this issue previously, in showing similar electrophysiological responses to NMDA in cortical neurons in the two culture systems, suggesting similar expression of NMDA receptors. However, the strongest argument against the criticism that the history of the cultures produces an intrinsic difference in vulnerability to excitotoxicity is that the difference in vulnerability observed disappears when a nontransported agonist such as NMDA is used or when glutamate uptake is blocked (sodium-free medium). This argues that the difference is due to the glutamate uptake system, and not to an intrinsic difference in vulnerability that might be generated by differing culture conditions.

The fact that uptake appears to play an important role in the protection of neurons in the astrocyte-rich cultures requires that at least some portion of the neurons be isolated from direct exposure to the medium, since uptake would not be expected to be able to diminish the medium concentrations of agonists quickly enough to account for all of the protection observed. It is noteworthy, however, that some evidence has been obtained suggesting that astrocytes may lower glutamate concentrations in unstirred layers above them in culture (Sugiyama et al., 1989). Since excitatory synapses are found primarily on dendrites ( $\mathrm{Pe}$ ters et al., 1991), these would be likely to be the most sensitive and vulnerable element of the neuron to stimulation by excitatory agonists. Since neuronal processes are difficult to discern in the astrocyte-rich cultures, it is likely that they are buried under the astrocytes in this culture system. This prediction has been borne out in electron microscopic studies in the astrocyterich cultures (K. M. Harris and P. A. Rosenberg, unpublished observations). In contrast, neuronal processes in astrocyte-poor cultures are directly exposed to the medium.

Taken together, these observations argue for an important role for astrocytes in modulating the vulnerability of cortical neurons to glutamate toxicity, and specifically that sodium-dependent glutamate uptake is protective of neurons in vitro as well as in vivo (Coyle et al., 1981; Mangano and Schwarcz, 1983). By implication, it is likely that compromise of astrocyte function by a variety of pathogenic mechanisms plays an important role in glutamate receptor-mediated neuronal injury and death.

\section{References}

Benjamin AM, Quastel JH (1976) Cerebral uptakes and exchange diffusion in vitro of $\mathrm{L}$ - and D-glutamates. J Neurochem 26:431-441.

Bottenstein JE, Sato GH (1979) Growth of a rat neuroblastoma cell line in serum-free supplemented medium. Proc Natl Acad Sci USA 76:514-517.

Bradford MM (1976) A rapid and sensitive method for the quantitation of microgram quantities of protein utilizing the principle of protein-dye binding. Anal Biochem 72:248-254.

Brew H, Attwell D (1987) Electrogenic glutamate uptake is a major current carrier in the membrane of axolotl retinal glial cells. Nature 327:707-709.

Choi DW (1987) Ionic dependence of glutamate neurotoxicity. J Neurosci 7:369-379.

Choi DW, Rothman SM (1990) The role of glutamate neurotoxicity in hypoxic-ischemic neuronal death. Annu Rev Neurosci 13:171182.

Choi DW, Koh J-Y, Peters S (1988) Pharmacology of glutamate neurotoxicity in cortical cell culture: attenuation by NMDA antagonists. J Neurosci 8:185-196.

Choi DW, Viseskul V, Amirthanayagam M, Monyer H (1989) Aspartate neurotoxicity on cultured cortical neurons. J Neurosci Res 23: 116-121.

Coyle JT, Bird SJ, Evans RH, Gulley RL, Nadler JV, Nicklas WJ, Olney JW (1981) Excitatory amino acid neurotoxins: selectivity, specificity, and mechanisms of action. Neurosci Res Prog Bull 19:331-427.

Drejer J, Larsson OM, Schousboe A (1982) Characterization of L-glutamate uptake into and release from astrocytes and neurons cultured from different brain regions. Exp Brain Res 47:259-269.

Drcjer J, Benveniste H, Diemer NH, Schousboe A (1985) Cellular origin of ischemia-induced glutamate release from brain tissue in vivo and in vitro. $\mathrm{J}$ Neurochem $45: 145-151$.

Faden AI, Demediuk P, Panter SS, Vink R (1989) The role of excitatory amino acids and NMDA receptors in traumatic brain injury. Science 244:798-800.

Garthwaite J (1985) Cellular uptake disguises action of L-glutamate on $N$-methyl-D-aspartate receptors. Br J Pharmacol 85:297-307.

Gordon RD, Balazs R (1983) Characterization of separated cell types from the developing rat cerebellum: transport of glutamate and aspartate by preparations enriched in Purkinje cells, granule neurons, and astrocytes. J Neurochem 40:1090-1099.

Koh J-Y, Choi DW (1988) Vulnerability of cultured cortical neurons to damage by excitotoxins: differential susceptibility of neurons containing NADPH-diaphorase. J Neurosci 8:2153-2163.

Mangano RM, Schwarcz R (1983) Chronic infusion of endogenous 
excitatory amino acids into rat striatum and hippocampus. Brain Res Bull 10:47-51.

Mattson MP, Guthrie PB, Kater SB (1989a) A role for $\mathrm{Na}^{+}$-dependent $\mathrm{Ca}^{2+}$ extrusion in protection against neuronal excitotoxicity. FASEB J 3:2519-2526.

Mattson MP, Murrain M, Guthrie PB, Kater SB (1989b) Fibroblast growth factor and glutamate: opposing roles in the generation and degeneration of hippocampal neuroarchitecture. J Neurosci 9:37283740 .

Meldrum B, Garthwaite J (1990) Excitatory amino acid neurotoxicity and neurodegenerative disease. Trends Pharmacol Sci 11:379-387.

Olverman HJ, Joncs AW, Mcwett KN, Watkins JC (1988) Structurc/ activity relations of $N$-methyl-D-aspartate receptor ligands as studied by their inhibition of $\left[{ }^{3} \mathrm{H}\right]_{\mathrm{D}}$-2-amino-5-phosphonopentanoic acid binding in rat brain membranes. Neuroscience 26:17-31.

Patneau DK, Mayer ML (1990) Structure-activity relationships for amino acid transmitter candidates acting at $N$-methyl-D-aspartate and quisqualate receptors. J Neurosci 10:2385-2399.

Peters A, Palay SL, Webster HD (1991) The fine structure of the nervous system. New York: Oxford UP.
Pixley SK, Cotman CW (1985) Laminin supports short-term survival of rat septal neurons in low-density, serum-free cultures. J Neurosci Res 15:1-17.

Rosenberg PA (1988) Catecholamine toxicity in cerebral cortex in dissociated cell culture. J Neurosci 8:2887-2894.

Rosenberg PA (1991) Accumulation of extracellular glutamate and neuronal death in astrocyte-poor cortical cultures exposed to glutamine. Glia 4:91-100.

Rosenberg PA, Aizenman E (1989) Hundred-fold increase in neuronal vulnerability to glutamate toxicity in astrocyte-poor cultures of rat cerebral cortex. Neurosci Lett 103:162-168.

Sugiyama K, Brunori A, Mayer ML (1989) Glial uptake of excitatory amino acids influences neuronal survival in cultures of mouse hippocampus. Neuroscience 32:779-791.

Tecoma ES, Monyer H, Goldberg MP, Choi DW (1989) Traumatic neuronal injury in vitro is attenuated by NMDA antagonists. Neuron 2:1541-1545.

Walicke P, Varon S, Manthorpe M (1986) Purification of a human red blood cell protein supporting the survival of cultured CNS neurons, and its identification as catalase. J Neurosci 6:1114-1121. 